\title{
Nanoscale membrane curvature sorts lipid phases and alters lipid diffusion
}

\author{
Xinxin Woodward and Christopher V. Kelly* \\ Department of Physics and Astronomy, Wayne State University, Detroit, MI, U.S.A. \\ *Corresponding author. Email: cvkelly@wayne.edu
}

\begin{abstract}
Cellular homeostasis requires the precise spatial and temporal control of membrane shape and composition. Membrane regions of high curvature, such as endocytic pits and viral buds, contain distinct lipids and proteins. However, the interplay between membrane curvature and local membrane composition is poorly understood at the nanoscale. Here, we employed single-molecule localization microscopy to observe single-lipid diffusion in model bilayers with varying lipid compositions, phase, temperature, and membrane curvature. Engineered membrane buds were observed for the creation of lateral compositional heterogeneity in otherwise homogeneous membranes. Membrane curvature recruited liquid-disordered lipid phases in phase-separated membranes and altered the diffusion of the lipids. Supported lipid bilayers were created over 50-nm radius nanoparticles to engineer nanoscale membrane curvature that mimics the size of naturally occurring endocytic pits and viral buds. The disorderpreferring lipids sorted to the nanoscale curvature at all temperatures, but only when embedded in a membrane capable of sustaining liquid-liquid phase separation at low temperatures. This result suggests that lipid sorting by the membrane curvature was only possible when coupled with lipid phase separation. The curvature affected the local membrane composition most strongly when the curvature was locally surrounded by a liquid-ordered phase typically associated with a stiffer bending modulus. The curvature-induced sorting of lipid phases was quantified by the sorting of disorder-preferring fluorescent lipids, single-lipid diffusion measurements, and simulations that couple the lipid phase separation to the membrane shape. Unlike single-component membranes, lipids in phase-separated membranes demonstrated faster diffusion on curved membranes than the surrounding, flat membrane. These results demonstrate that curvature-induced membrane compositional heterogeneity can be achieved by collective behavior with lipid phase separation when single-molecule properties (i.e., packing parameter) are insufficient. These results support the hypothesis that the coupling of lipid phases and membrane shape may yield lateral membrane composition heterogeneities with functional consequences.
\end{abstract}

\section{STATEMENT OF SIGNIFICANCE}

Nanoscopic membrane organization and dynamics are critical for cellular function but challenging to experimentally measure. This work brings together super-resolution optical methods with engineered substrates to reveal the interplay between curvature, composition, phase, and diffusion in model membranes. We report that curvature can induce phase separation in otherwise homogeneous membranes and that the phase-curvature coupling has a direct implication on lipid mobility. In sum, this discovery advances our understanding of the fundamental membrane biophysics that regulate membrane activities such as endocytosis and viral budding.

\section{KEYWORDS}

Membrane curvature; phase separation; single-particle tracking; molecular sorting; and diffusion. 


\section{1) INTRODUCTION}

Live-cell plasma membranes contain hundreds of lipid species that can separate into coexisting liquid phases (1-3). Phase separated domains are thought to be critical for cell functions such as protein sorting, cell signaling, membrane budding, and retrovirus replication (4-7). Caveolae, for example, are cholesterol and sphingolipid-rich endocytic buds that have diverse roles in membrane compositional regulation, force sensing, and endocytosis $(8,9)$.

Model membranes of known lipid mixtures provide a means to reveal how the membrane composition affects its function. Coexisting liquid-ordered $\left(L_{0}\right)$ and liquid-disordered $\left(L_{d}\right)$ phases are created by combining a sterol with a phospholipid with a high melting temperature and a phospholipid with a low melting temperature below their miscibility transition temperature $\left(T_{m}\right)(10-13)$. Phase-separated giant unilamellar vesicles (GUVs) and supported lipid bilayers (SLBs) have shown curvature-dependent phase separations connected with the difference in bending rigidity of the $L_{d}$ and $L_{o}$ phases (14-19). In model membranes, the region of greater curvature concentrates the disordered phase $(18,19)$. However, endocytic processes and viral budding in live cells are typically correlated with $L_{o}$-preferring lipids, e.g., cholesterol and sphingomyelin. The detailed lipid diffusion, partitioning coefficient, and temperature dependency of real and model membranes remain unknown and fundamental to the membrane biophysics in live cells. This manuscript aims to advance our understanding of curvature on model membranes as a critical step to understanding complex living cell membranes.

Lipid diffusion in the $L_{d}$ phase is up to $10 x$ faster than lipids in the $L_{o}$ phase, depending on the membrane composition, the fluorescent lipids tracked, and the substrate topography (13, 20-23). For example, DPPE-Texas Red in SLBs composed of DiPhyPC, DPPC, and cholesterol at molar ratios of 2:2:1 had a diffusion coefficient of $1.8 \pm 0.5$ greater in the $L_{d}$ phase than the $L_{o}$ phase (24). The diffusion in the two phases becomes more similar to increasing the temperature or increasing cholesterol content, as is correlated with shorter tie-lines separating the phases. SLBs composed of DiPhyPC, DPPC, and cholesterol at molar ratios of 1:1:2 demonstrated lipid phase separation, as evident by the partitioning of DPPE-Texas Red, but no difference in DPPE-Texas Red diffusion was observed in the two phases (24).

The influence of membrane curvature on single-lipid diffusion has been studied in welldefined geometries. Diffusion typically slows on cylindrical membranes when the radii are smaller than $50 \mathrm{~nm}$ (25). Some fluorescent lipids slow on spherical membranes with a $50-\mathrm{nm}$ radius $(26,27)$. The effects of curvature on lipid diffusion are correlated to the fluorophore location on the labeled lipid; head-group-labeled lipids diffuse $3 x$ faster on flat versus curved membranes, whereas tail-labeled lipids diffuse at the same rate on flat and curved membranes (26).

In this manuscript, the diffusion and sorting of lipids relative to nanoscale membrane curvature are quantified. Membrane curvature was generated by creating phase-separated SLBs over 50-nm radius nanoparticles on planar microscopy coverslips (Fig. 1). The concentration of DPPE-Texas Red on the curved membrane was measured via diffractionlimited images and analyzed for lipid sorting. Single-particle tracking (SPT) was performed to track DPPE-Texas Red diffusion and to measure the curvature-dependent lipid mobility. The lipid phase was more disordered for the curved membrane than the surrounding planar bilayer, regardless of the phase of the surrounding bilayer. However, the disorder-preferring lipids sorted to the curvature more strongly if the immediately surrounding planar membrane was more ordered. The single-lipid diffusion was up to $2.2 x$ faster on curved versus planar 
membranes, with more significant differences observed when the tie-lines were longer. These experimental results were verified by applying a computational simulation of phase separation that incorporated phase-curvature coupling. Both the sorting of disordered lipids to the curvature and the effects of the curvature propagating onto the surrounding planar membrane were observed experimentally and computationally. This study provides a further understanding of the relationship between phase separation and membrane curvature at a physiological length scale. These results show that lipid phase sorting coupled to nanoscale membrane bending manifests in molecular sorting and single-molecule diffusion correlated with membrane curvature.

\section{2) MATERIALS AND METHODS}

\section{1) GUV formation}

The lipids diphytanoylphosphatidylcholine (DiPhyPC), dioleoylphosphatidylcholine (DOPC), dipalmitoylphosphatidylcholine (DPPC), palmitooleoylphosphatidylcholine (POPC), and cholesterol (Avanti Polar Lipids) were used without further purification (Fig. 1A). Fluorescent dihexadecanoylphosphoethanolamine-Texas Red (DPPE-Texas Red, Life Technologies) was included at 0.1 mol\% for labeling for all membranes with DiPhyPC or POPC. Top Fluorpalmitoylundecanoylphosphatidylcholine (TopFluor-PC; Avanti Polar lipids) was included at 0.2 mol\% in membranes with DOPC. Milli-Q water with a resistivity of $18 \mathrm{~m} \Omega$ was used in all buffers. All other chemicals were purchased from Sigma Aldrich.

GUVs were created by electroformation, as described previously (28). Briefly, lipids were combined in chloroform and dried onto conducting indium tin oxide-coated glass plates. A trimmed silicon sheet was added between the plates to form the incubation chamber. The chamber was filled with a $200 \mathrm{mM}$ sucrose solution and transmitted an $\mathrm{A}_{\mathrm{C}}$ signal with $\mathrm{V}_{\mathrm{rms}} 3 \mathrm{~V}$ at $10 \mathrm{~Hz}$ for $1 \mathrm{hr}$ at $55^{\circ} \mathrm{C}$. The GUV solution had $13 \mathrm{mg}$ of lipids per $\mathrm{mL}$ after electroformation. GUVs were stored at $55^{\circ} \mathrm{C}$ and used within 2 days.

\section{2) Sample dish preparation}

Glass-bottom dishes (MatTek Corp.) were initially rinsed with ethanol, blown dry by a nitrogen stream, then placed in air plasma (Harrick Plasma) for $10 \mathrm{sec}$ to create a hydrophilic surface. $20 \mu \mathrm{L}$ of $5 \mathrm{mM} \mathrm{CaCl}_{2}$ was spun on a glass substrate at $100 \mathrm{rpm}$. $50-\mathrm{nm}$ radius fluorescent nanoparticles that were excited at $405 \mathrm{~nm}$ (Fluoro-Max; Fisher Scientific) were used for generating curvature, and multicolored nanoparticles (TetraSpeck, ThermoFisher Scientific Technologies) were used as stage drift correction fiducial marks. The nanoparticle-containing solutions were evaporated from the coverslips by a hot plate at $35^{\circ} \mathrm{C}$ for $5 \mathrm{~min}$. The dishes were then chilled to room temperature prior to GUV deposition.

\section{3) Supported lipid bilayer formation}

SLBs with engineered curvature were created by GUV fusion over nanoparticles on microscopy coverslips. The GUV solution was placed in the refrigerator at $4{ }^{\circ} \mathrm{C}$ for 2 minutes to assist the macro-domain formation. $5 \mu \mathrm{L}$ of the chilled GUV solution was applied to the room temperature glass bottom dishes with nanoparticles. $50 \mu \mathrm{L}$ of $4^{\circ} \mathrm{C}$ Milli-Q water was added to encourage the GUVs to sink to the cover glass. The dish was chilled to $4^{\circ} \mathrm{C}$ for 15 min before gentle rinsing with $5 \mathrm{~mL}$ of $4^{\circ} \mathrm{C}, 200 \mathrm{mM}$ sucrose. The creation of SLBs over nanoparticles for engineered curvature has been performed previously $(26,27,29-31)$.

\section{4) Imaging procedure}


The optical setup included an inverted IX83 microscope with a 100x, 1.49 NA objective (Olympus), a 2x emission path magnification (OptoSplit, Cairn Research), and an iXon 897-Ultra EMCCD camera (Andor Technology), as described previously (26, 30). A Hg lamp with an excitation filter (BrightLine single-band filters, Semrock) provided illumination for diffractionlimited images. CUBE diode lasers with wavelengths of 405 and $488 \mathrm{~nm}$ (Coherent) and a 561 $\mathrm{nm}$ Sapphire laser (Coherent) were used for single-molecule localization microscopy. The excitation light passed through a clean-up filter (zet405/488/561/647x, Chroma Technology), encountered a quad-band dichroic mirror (zt405/488/561/647rpc, Chroma Technology), and reflected into the objective. The emission was isolated via emission filters (BrightLine singleband filters, Semrock) and a 4-band notch filter (zet405/488/561/640m, Chroma Technology). The SOLIS imaging software (Andor Technology) was used to acquire images with 128 pixels $x$ 128 pixels region of interest in the kinetic mode and an EM gain of 150. The images were acquired at $537 \mathrm{~Hz}$. Typically, 20,000 frames were combined to create reconstructed, superresolution images or analyzed for single-particle tracking.

\section{5) Membrane temperature control}

A Peltier temperature control dish holder (QE-1HC, Warner Instruments) was used with a custom, insulated dish cover with a thermocouple mount. Once the dish cover is placed on the sample dish, the thermocouple was $<0.5 \mathrm{~mm}$ above the center of the glass coverslip. The temperature was actively controlled via a custom LabVIEW program. Also, the dishes were never heated above $45^{\circ} \mathrm{C}$ to protect the microscope objective. When changing temperature, the temperature was changed at $0.5^{\circ} \mathrm{C} / \mathrm{min}$ and remained at the set temperature for 30 min before imaging. The Peltier dish holder was initially set to $10^{\circ} \mathrm{C}$ before inserting a membrane sample. The Peltier temperature was set to be at $10^{\circ} \mathrm{C}, 30^{\circ} \mathrm{C}$, and $45^{\circ} \mathrm{C}$ resulted in the thermocouple measuring a temperature of $17 \pm 3^{\circ} \mathrm{C}, 27 \pm 1^{\circ} \mathrm{C}$, and $37 \pm 1^{\circ} \mathrm{C}$, respectively.

\section{6) Lipid phase identification}

The 2D projected image of a homogenous membrane yields an increased brightness at the location of membrane curvature compared to the surrounding planar membrane due to the increase in the projected membrane area. Coincident nanoparticles confirmed the membrane curvature in the appropriate color channel. A single 2D Gaussian fit was used to find the center of membrane curvature in the membrane color channel.

The lipid phase was quantified by the partition coefficient of the fluorescent lipids. Both DPPE-Texas Red and TopFluor-PC are $L_{d}$ preferring lipids $(32,33)$, and the higher local concentration was interpreted as a greater degree of disorder in the acyl tails. The intensity of the curved membrane $\left(I_{C}\right)$ is defined as the average intensity of the nine pixels within $170 \mathrm{~nm}$ from the center of curvature, and the intensity surrounding the curvature $\left(I_{F}\right)$ is defined as the average intensity of the pixels $400 \pm 56 \mathrm{~nm}$ away from curvature from diffraction-limited images (Fig. S1). The increased membrane brightness due to the membrane topography was determined from homogeneous POPC membranes and analytical models of the membrane shape, which provide the expected membrane curvature intensity increase due to the increased membrane area in the $\mathrm{XY}$-projection, $I_{C}^{P O P C} / I_{F}^{P O P C}$. The average membrane intensity $(<\mid>)$ is defined as the midpoint of the mean $L_{d}$ phase intensity $\left(I_{d}\right)$ and mean $L_{o}$ phase intensity $\left(I_{o}\right)$ from each planar SLB patch; $\langle l\rangle=\left(l_{d}+I_{0}\right) / 2$. This definition of $\langle>$ accounts for individual fields of view that happened to image more of one phase than the other. When no phase separation was present on the flat membrane, $\langle l\rangle=I_{F}$. The lipid phase on the flat membrane surrounding the curvature $\left(P_{F}\right)$ and the phase of the curvature $\left(P_{C}\right)$ was determined by

$$
P_{F}=\frac{I_{F}}{<I>}
$$




$$
P_{C}=\frac{I_{C}}{<I>} \frac{I_{F}^{P O P C}}{I_{C}^{P O P C}}
$$

This method of determining the lipid phase from fluorescence images accounts for sample-tosample variation in fluorophore concentrations and temporal changes caused by fluorescence bleaching.

If the membrane was homogeneous or there was no correlation between the lipid phase and the membrane curvature, then expected $P_{F}$ and $P_{C}$ would both equal 1 . The magnitude of the phase sorting to curvature was quantified based on how different the phase on the curved membrane was compared to the surrounding flat membrane, i.e., $P_{C} / P_{F}$. The ratio of $P_{C} / P_{F}$ is analogous previously used quantifications of phase sorting (15) and to a curvature-dependent partition coefficient. To measure the phase- and curvature-dependent lipid diffusion, individual membrane curvature events were grouped based on the phase of the surrounding flat membrane. The individual curvature events were deemed $L_{d}$ or $L_{o}$ phase if $P_{F}$ was greater than 1.1 or less than 0.9 , respectively.

Prior reports vary with labeling the warm, homogeneous liquid phase of mixed lipids as $L_{o}(34,35)$ or leaving it unlabeled $(36,37)$. Within this manuscript, we use the label $L$ as a representation of its intermediate qualities of this homogenous, multicomponent liquid phase while reserving the label of $L_{\alpha}$ for the liquid phase for a single-component membrane.

\section{7) Single-fluorophore localization}

The movies with optically isolated fluorescent lipids were analyzed by the Fiji plug-in ThunderSTORM, which fit every single-fluorophore image with a 2D Gaussian function to export its location, intensity, fit width, and fit certainty $(38,39)$. Only the localizations with intensity > 100 photons, Gaussian fit width $>15 \mathrm{~nm}$, and location uncertainty < $45 \mathrm{~nm}$ were kept for further analysis. The ThunderSTORM-reported fluorophore uncertainty was $24 \pm 1 \mathrm{~nm}$. The stage drift during imaging was corrected by analyzing the motion of the multicolored nanoparticles through built-in ThunderSTORM fiducial tracking, which was separate from the nanoparticles used to engineering membrane curvature.

\section{8) Super-resolved curvature identification and aggregate removing method}

Reconstructed super-resolution images of single-lipids revealed increased localization density at the sites of membrane curvature. Curvature was confirmed by the colocalization of nanoparticles in the complementary color channel. The local density increase in single-lipid localizations was fit to a 2D Gaussian function to find the exact curvature center for the superresolution images and single-particle tracking analysis.

DiPhyPC-containing SLBs yield aggregates of DPPE-Texas Red (24). Aggregates were apparent via a high fluorophore localization density in the absence of nanoparticles.

Comparisons between membrane curvature events and fluorophore aggregates were made via the size of the cluster of localizations, the local single-lipid diffusion rate, localization density, localization rate, and the proximity to the nanoparticles, as detailed in the Discussion. Aggregates were removed via localization density and long trajectory culling, as described previously (24). Briefly, all localizations that were not associated with membrane curvature were grouped based on their 2D location and acquisition time into 3D voxels. When the number of localizations in a voxel exceeded the localization density threshold $\left(\rho_{t h}\right)$, that region of the sample was deemed to be an aggregate and all localizations within that $\mathrm{XY}$-region were excluded from subsequent analysis. Further, all localizations associated with trajectories that 
lasted more than 32 steps were culled. A more detailed description of the localization culling to remove aggregates is given in the Supplemental Material.

\section{9) Single-particle tracking}

The single-lipid localizations were linked via u-track with a maximum step length of 400 $\mathrm{nm}$ (40). The average trajectory length of the aggregate-removed localizations was $6 \pm 4$ steps. Single-lipid step lengths $(v)$ were grouped versus their distance away from the center of curvature $(r)$ and fit to a 2D Rayleigh distribution $(R)(26,41,42)$,

$$
R(v)=\frac{v}{2 D_{f i t} \Delta \mathrm{t}} e^{-\frac{v^{2}}{4 D_{f i t} \Delta \mathrm{t}}}
$$

Fitting of Eq. 3 incorporates the time between sequential frames $(\Delta t)$ and yields a fit diffusion coefficient $\left(D_{\text {fit }}\right)$. $D_{\text {fit }}$ was corrected for imaging blur created by the single-frame exposure time $\left(t_{\text {exp }}\right)$ and localization uncertainty $\left(\sigma_{r}\right)$ through Eq. 4 (43-45),

$$
D_{x y}=\frac{D_{f i t}-\frac{\sigma_{r}^{2}}{2 \Delta t}}{1-\frac{t_{e x p}}{3 \Delta t}} .
$$

The resulting diffusion coefficient through the $X Y$-plane $\left(D_{x y}\right)$ requires further correction to account for the 3D membrane shape. Monte Carlo simulations were performed for random walk on the surface that approximates the membrane bending over the $50-\mathrm{nm}$ radius nanoparticle, as done previously $(24,26,30,46)$. These simulations incorporated a varying lipid diffusion rate on the planar versus curved membrane and were analyzed to mimic the experimental conditions with an imposed localization uncertainty and image blur. The simulations show that $D_{x y}(r \leq 50 \mathrm{~nm})$ underestimates the in-membrane diffusion by a factor of $0.53 \pm 0.10$ due to the membrane tilt relative to the XY-plane for membranes wrapping the 50$\mathrm{nm}$ radius nanoparticles. Accordingly, the diffusion of the planar $\left(D_{F}\right)$ and curved $\left(D_{C}\right)$ membranes is approximated by

$$
\begin{aligned}
& D_{\text {Flat }}=D_{x y}(400 \mathrm{~nm} \leq r \leq 600 \mathrm{~nm}) \\
& D_{\text {Curved }}=D_{x y}(r \leq 50 \mathrm{~nm}) / 0.53
\end{aligned}
$$

\subsection{0) Simulation of phase-curvature coupling}

Lipid phase separation and phases-curvature coupling was modeled with a Hamiltonian consisting of three parts: membrane height fluctuations via the Helfrich form; local compositional fluctuations via a fourth-order Landau expansion; and curvature-composition coupling (47),

$$
H=\int\left[\kappa\left(\nabla^{2} h\right)^{2}+\sigma(\nabla h)^{2}+A \varphi^{2}+B(\nabla \varphi)^{2}+C \varphi^{4}-\gamma \varphi\left(\nabla^{2} h\right)\right] d x d y .
$$

The Hamiltonian is dependent on the membrane height $(h)$, bending rigidity $(\kappa)$, the surface tension $(\sigma)$, phase $(\varphi)$, Landau phase constants $(A, B$, and $C)$, and phase-curvature coupling strength $(\gamma)$. Our simulation examined the distribution of lipid phases on the membrane of fixed curvature, similar to as done experimentally. Both $\kappa$ and $\sigma$ were set to zero because $h$ was constant. The Landau phase constants $A, B$, and $C$ enable modeling the phase separation away from the critical point where first-order phase transitions are expected. We set $A=-1, B=0.7$, 
and $\mathrm{C}=4$ that minimize $H$ for flat membranes (i.e., $\nabla^{2} h=0$ ) when $\varphi= \pm 2^{-3 / 2}$ when the domains are well separated (i.e., small $\left.(\Delta \varphi)^{2}\right)$. Positive values of $\gamma$ sorted positive values of $\varphi$ to the curvature, which we labeled as $L_{d}$. A time step of our simulation included minimizing $H$ by small changes to $\varphi$ equal to $-0.05^{*} \mathrm{~d} H / \mathrm{d} \varphi$ at each location of the membrane. Thermal fluctuations were modeled as the addition of a normal distribution of random perturbations to $\varphi$. The standard deviation of this noise distribution $(\sigma)$ corresponds to temperature $(T)$, which was calibrated by qualitative matching of the experimental observations; flat bilayers displayed no stable phase separation at $T=35^{\circ} \mathrm{C}(\sigma=0.08)$ and large domains at $T=15^{\circ} \mathrm{C}(\sigma=0.07)$. Additionally, the perimeter of each simulation $(r \geq 150 \mathrm{~nm}$ ) was rescaled each time step to have $a\langle\varphi\rangle=0$ to prevent the simulation from becoming a single large domain of one phase at low temperatures. Movies of the phase dynamics and a static image of the average phase separation on flat and curved membranes are provided in the Supporting Information (Movie S1 and Fig. S3).
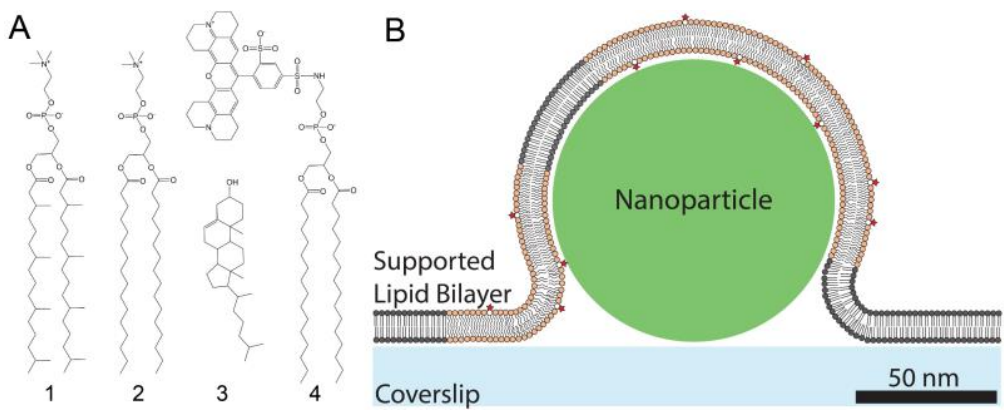

Figure 1: Phase-separated supported lipid bilayers included mixtures of lipids draped over a 50-nm radius nanoparticle. (A) The lipids most frequently used in this study include (1) DiPhyPC, (2) DPPE, (3) cholesterol, and (4) DPPE-Texas Red. (B) The $L_{d}$ (yellow) and $L_{o}$ (gray) lipid phases are analyzed relative to the engineered, nanoparticle-induced membrane curvature.

\section{3) RESULTS}

\section{1) Phase separation observed in SLBs}

To track the lipid phase separation within the membranes, we added fluorescence tracer lipids to ternary mixtures of DiPhyPC, DPPC, and cholesterol. The phase-separated SLBs had bright regions indicating the $L_{d}$ phase, dim regions indicating the $L_{o}$ phase, and black regions indicating the lack of an SLB over the coverslip (Fig. 2). The fluorescence contrast between the $L_{o}$ and $L_{d}$ phases reflects the partition coefficient of the fluorescent lipid and the length of the tieline that separates the two phases. The $L_{o}$ and $L_{d}$ phases were more similar in brightness at higher cholesterol concentrations and at higher temperatures.

The $T_{m}$ for SLBs with a molar ratio of 1:1:2 DiPhyPC:DPPC:cholesterol was between 28 and $37^{\circ} \mathrm{C}$, as evident by the stable phase separation at $28^{\circ} \mathrm{C}$ and the phase mixing that occurred at $37^{\circ} \mathrm{C}$. All samples were equilibrated for $>30$ min prior to measurements of phase separation or lipid diffusion at a given temperature. The slow large-scale mixing of SLBs resulted in some of the largest domains (>10 $\mu$ m diameter) remaining evident with blurred phase boundaries even when the sample temperature was greater than $T_{m}$, as described previously $(24,48)$. The $T_{m}$ for $2: 2: 1 \mathrm{SLBs}$ was greater than $37^{\circ} \mathrm{C}$, as evident by the clear phase separation observed at this temperature. Varying the sample temperature enabled testing the effects of varying the tie-line length on lipid sorting and diffusion. 


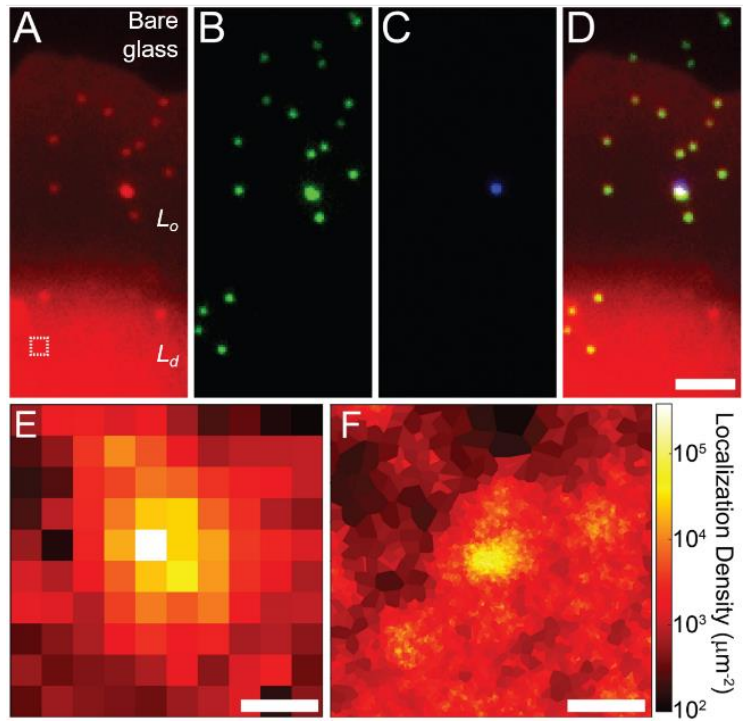

Figure 2: Phase-separated SLBs were created with nanoscale membrane curvature from a 1:1:2 molar ratio of DiPhyPC:DPPC:cholesterol and $0.1 \mathrm{~mol} \%$ DPPE-Texas Red. Diffraction-limited fluorescence images include those of (A,E) DPPE-Texas Red, (B) 50-nm radius fluorescent nanoparticles, (C) multicolored nanoparticle fiducial markers, and (D) their merge. (A) The phase-separated SLB contained a micron-scale $L_{d}$ domain (bright, bottom) and $L_{o}$ domain (dim, middle) while surrounded by bare coverslip (black, top). Nanoscale puncta represent an increased membrane area and a sorting of the $L_{d}$ phase where membrane curvature is present, such as the outlined region of $(A)$ that is shown as a zoom-in (E). (F) A Voronoi image of an SLB with engineered curvature reveals $20-\mathrm{nm}$-resolution in a region analogous to that shown in (E). (A-D) Scale bar, $2 \mu \mathrm{m}$. (E,F) Scale bar, $200 \mathrm{~nm}$.

\section{2) $L_{d}$-preferring lipids sort to curvature}

To test the hypothesis that nanoscale membrane curvature preferentially recruited disordered lipid phases, we engineered curvature in SLBs by bursting phase-separated GUVs upon 50-nm radius nanoparticles and glass coverslips (Figs. 1,2). This setup provided a constant membrane topography while allowing individual lipids to diffuse freely within the SLB The connectivity of the membrane between the planar SLB and the membrane bud over the nanoparticle was demonstrated both with fluorescence recovery after photobleaching and the observation of uninterrupted of single-lipid trajectories.

The membrane curvature induced lipid sorting with disorder-preferring lipids concentrating on the curvature (Fig. 3). The lipid phase on the curved membrane $\left(P_{C}\right)$ was compared to the phase of the flat membrane $\left(P_{F}\right)$ that was immediately surrounding the curvature. The curvature-dependent sorting of the $L_{d}$ phase is evident by $P_{C}$ being greater than $P_{F}$ for SLBs composed of DiPhyPC, DPPC, and cholesterol at a molar ratio of 1:1:2. The ratio $P_{C} / P_{F}$ was greater than 1 for all temperatures and all values of $P_{F}$, which indicates that the curved membrane was consistently more disordered than the surrounding planar membrane. The ratio $P_{C} / P_{F}$ was larger when $P_{F}$ was lower, which reveals that the curvature-induced sorting of lipid phases was strongest when the surrounding phase was more ordered. 

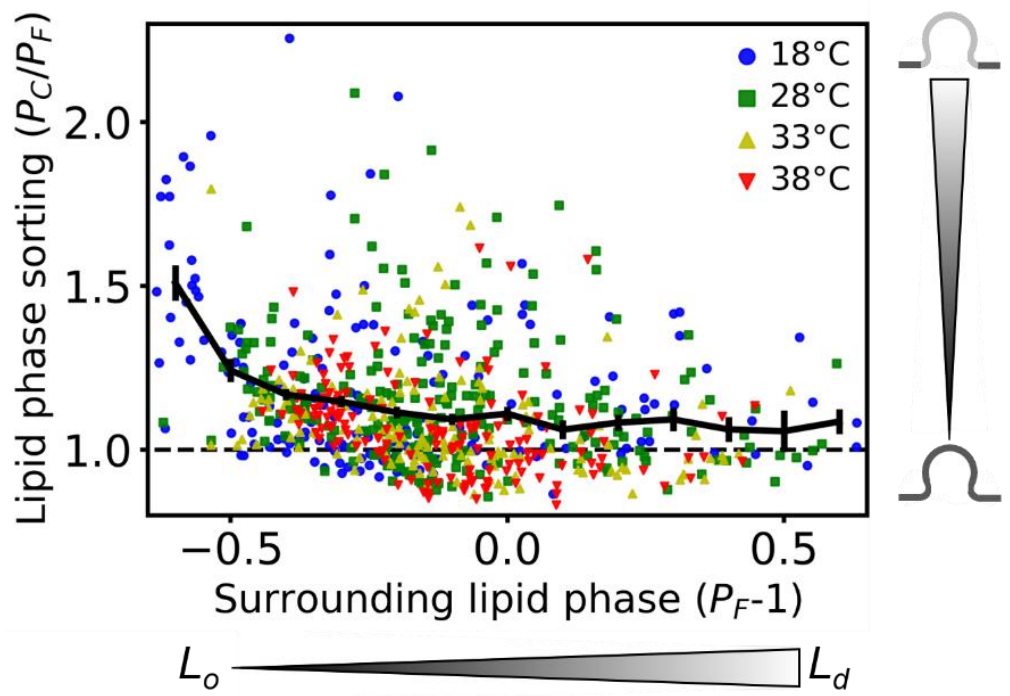

Figure 3: Lipids on curved membranes are more disordered than planar surrounding membrane (i.e., $P_{C} / P_{F}>$ 1). The curvature-dependent phase sorting was greatest when the phase surrounding the curvature is more ordered (i.e., $\left.\left(P_{F-1}\right)<0\right)$. Shown here are the curvature-induced phase sorting results for SLBs with a 1:1:2 molar ratio of DiPhyPC:DPPC:cholesterol at temperatures between 18 and $38^{\circ} \mathrm{C}$.

$P_{C}$ and $P_{F}$ were measured for varying membrane compositions and temperatures. Lower temperatures provide a greater range in $P_{F}$ values, consistent with lower temperatures providing longer tie-lines separating the phases. However, the analysis of $P_{C} / P_{F}$ versus $P_{F}$ demonstrates no variation with sample temperature, which reveals that the sorting of disordered lipids to the curvature was not temperature-dependent.

Curvature-induce lipid phase sorting was confirmed in SLBs made with a 7:7:6 molar ratio of DOPC:DPPC:cholesterol and labeled with the fluorescent lipid TopFluor-PPC, which concentrates in disordered lipid phases (Fig. S2). Again, $P_{C} / P_{F}$ was greater than 1, indicating that disorder-preferring lipids sorted to the curved membranes. Similarly, the curvature-induced sorting was most significant for nanoparticle-induced membrane curvature regions that were immediately surrounded by ordered lipids (i.e., $\left.\left(P_{F}-1\right)<0\right)$.

\section{3) Single-lipid diffusion on membrane curvature}

To test the hypothesis that the curvature-phase coupling results in a curvature-induced increase in lipid diffusion, SPT was performed to correlate the single-lipid dynamics with the membrane topography. The single-lipid trajectories were observed within SLBs relative to the engineered curvature with a 50-nm radius. The application of Rayleigh distribution fitting rather than the conventional mean-square displacement analysis provided a precise determination of the diffusion coefficient across the nanoscale membrane curvature $(24,26,46)$. The lipid trajectories were projected into the $\mathrm{XY}$-plane and binned according to their distance from the curvature-generating nanoparticle (Fig. 4).

Quasi-single component SLBs with no phase separation revealed variation in $D_{x y}$ over the nanoparticle due to both the curvature-dependent diffusion rates and the geometrical effects of projecting the 3D membrane trajectories into the $X Y$-plane for imaging. The contribution due to the $\mathrm{XY}$-projection was estimated from Monti-Carlo simulations to reveal an effective diffusion rate in the curved membrane, $D_{C}$, as described above and previously (26). Here, the lipid phase, curvature, and temperature-dependent diffusion of DPPE-Texas Red was measured. 

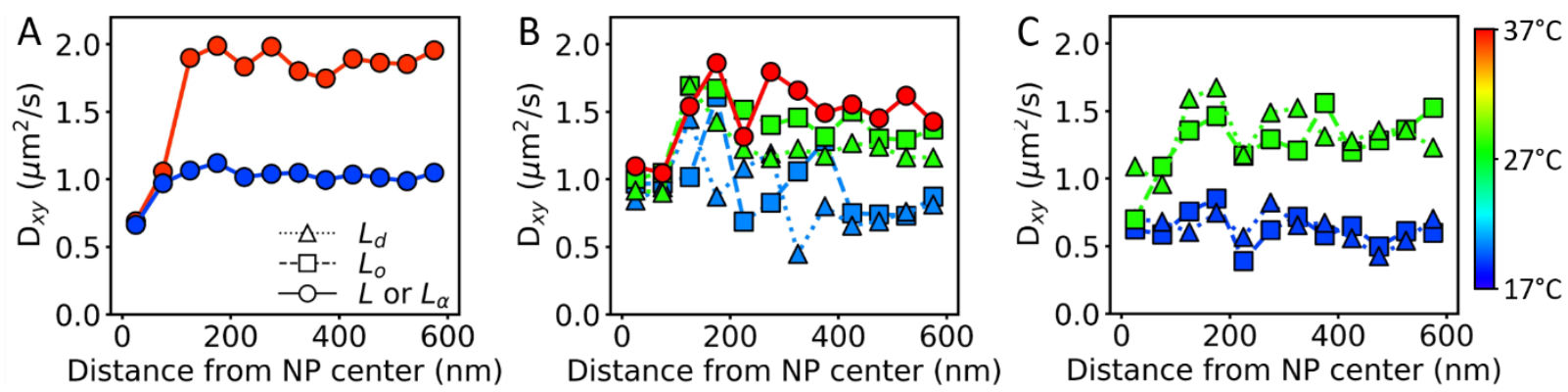

Figure 4: $D_{x y}$ as a function of lateral distance away from the nanoparticle $(N P)$ center for $(A)$ DiPhyPC SLBs and phase-separated SLBs composed of (B) 1:1:2 or (C) 2:2:1 molar ratios of DiPhyPC:DPPC:cholesterol. $D_{x y}$ was affected the membrane curvature, temperature, lipid phase, and the projection of the 3D membrane topography into the $\mathrm{XY}$-imaging plane.

Diffusion versus location on the membrane curvature locations was studied for SLBs of 1:0:0, 2:2:1, and 1:1:2 molar ratios of DiPhyPC:DPPC:cholesterol. The ratio of $D_{C} / D_{F}$ was consistently higher for systems with longer tie-lines separating the coexisting $L_{d}$ and $L_{o}$ lipid phases. The greatest $D_{C} / D_{F}$ ratio was observed in $20^{\circ} \mathrm{C}$ SLBs with a 1:1:2 molar ratio of DiPhyPC:DPPC:cholesterol in which $D_{C} / D_{F}=2.3 \pm 1.0$ (Fig. $3 \mathrm{C}$, Table S1). In contrast, $37{ }^{\circ} \mathrm{C} L_{\alpha}$ phase DiPhyPC SLBs displayed the smallest $D_{C} / D_{F}$ ratio equal to $0.4 \pm 0.1$.

As the temperature increased, $D_{F}$ increased, as seen previously $(24,49-51)$. The interplay of multiple effects resulted in $D_{C}$ for 1:1:2 SLBs not showing a difference with temperature change. Interestingly, $D_{x y}$ decreases from $125 \mathrm{~nm} \leq r<400 \mathrm{~nm}$ in ternary mixtures at lower temperatures and higher cholesterol content (Fig. 4). We hypothesize this change in $D_{x y}$ at these intermediate $r$ values may be due to curvature-seeded $L_{d}$ phase propagation onto the planar SLB surrounding the nanoparticle, which was replicated in simulations described below.

All values of $D_{C} / D_{F}$ for all ternary mixtures system are greater or equal to one, which indicates that that the lipids in phase-separated membrane curvature diffuse faster at curvature sites than flat (Fig. 4). $D_{C} / D_{F}$ was larger at the colder temperatures, indicating that $L_{d}$-preferring lipids sort to the curvature more when the tie-line separating the phases was longer. This was consistent with the diffraction-limited imaging of curvature-phase sorting (Fig. 2).

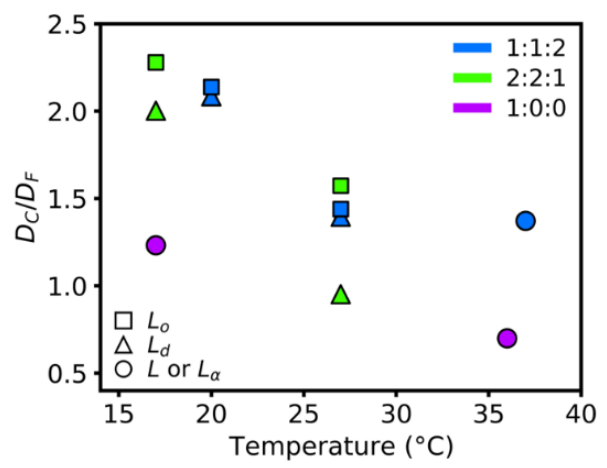

Figure 5: The diffusion ratio in curved versus flat membranes $\left(D_{C} / D_{F}\right)$ are shown for varying membrane composition, phase, and temperature. The color indicates the DiPhyPC:DPPC:cholesterol molar ratios and the marker shape indicates the lipid phase. Uncertainty levels for each data point are given in Table S1.

\section{4) Simulated lipid phase sorting by membrane curvature}


To test the consistency between our experimental data and established theoretical models of membrane phase separation coupled to curvature, we performed computational simulations. The membrane was modeled to have a topography consistent with the experimental, 50-nm radius, nanoparticle-supported SLBs (Fig. 6A). Landau phase constants were chosen to provide two stable phases with fluctuations in composition and phase boundary. The magnitude of a Gaussian noise profile modeled the thermal fluctuations and temperature of the membrane. At low temperatures, large single-phase domains formed (Fig. 6B). At high temperatures, rapid phase fluctuations resulted in no large-scale or stable phases present on the planar membrane (Fig. 6C). The Supplemental material includes movies of these simulations (Movie S1) and an image of the average phase during the simulation (Fig. S3). When the simulated membrane was at a lower temperature, the $L_{d}$ phase displayed greater sorting to the curvature. Further, the effects on curvature can propagate onto the surrounding planar bilayer (Fig. 6D).

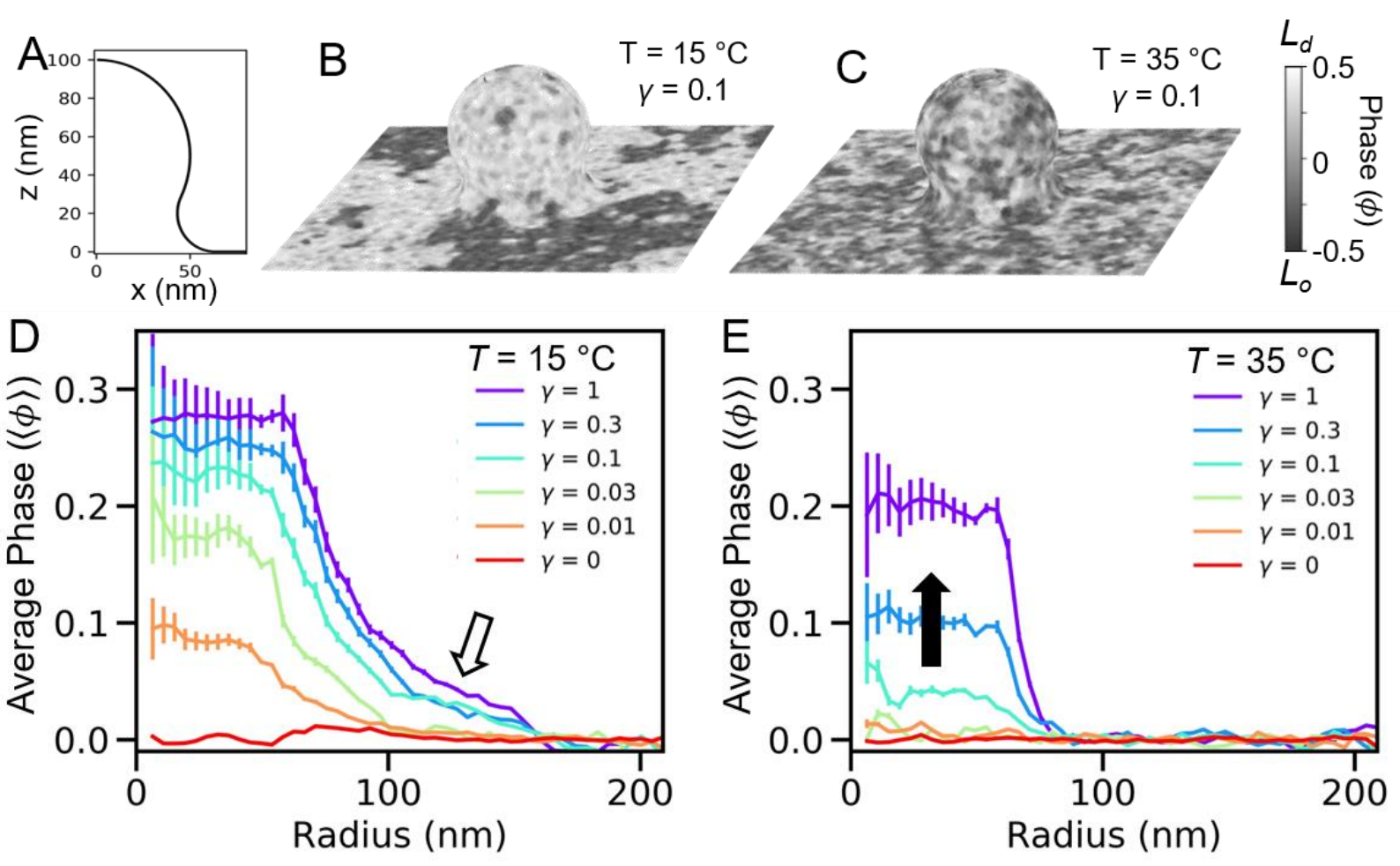

Figure 6: Phase-curvature coupling was reproduced in computational simulations. A phase-separated membrane was modeled with a fixed membrane geometry $(A)$ at a low $(B)$ or a high $(C)$ temperature and with varying phase-curvature coupling $(\gamma)$. (B, D) At low temperatures, the curvature-preferring $L_{d}$ phase spreads to the surrounding, flat membrane (white arrow). (C, E) At high temperatures, when no stable phase separation is observed on the flat membrane, the curved membrane may still be more disordered than the average membrane (black arrow).

\section{4) DISCUSSION}

This study provides a quantitative analysis of the phase-curvature coupling of SLBs with membrane shapes reminiscent of nanoscopic endocytic pits or viral buds. The composition, temperature, and curvature were varied for experimental and simulated membranes. The effects of curvature on the phase sorting were consistently more substantial with membrane conditions 
that provided a longer tie-line separating the phases, i.e., lower temperature and less cholesterol. The phase sorting was observed by both the lateral heterogeneity in the local density and the apparent diffusion coefficient of disorder-preferring fluorescent lipids. However, curvature-induced sorting and curvature-affected diffusion rates were apparent even when phase separation was not observed on the planar membrane. Accordingly, these results suggest that the nanoscale curvature and complex components of native biological membranes may yield significantly coupling between the membrane shape, composition, and effective viscosity.

\section{1) Diffraction-limited images yield curvature-induced sorting of lipids}

The degree of lipid sorting with curvature was quantified by measuring the fluorescence intensity difference between the engineered curvature and the surrounding planar membrane via diffraction-limited epifluorescence imaging. The membrane intensity in diffraction-limited images represents the fluorophore concentration with $200 \mathrm{~nm}$ resolution. Difffraction-limited imaging requires two orders of magnitude less illumination intensity than super-resolution imaging, which is important for reducing the light-induced phase separation. Also, our superresolution methods show only a few fluorophores at a frame and required $>20,000$ frames to study the diffusion. The single-lipid localizations measured relatively few molecules per reconstructed image with the localization rate dependent on the time since imaging began and the fluorophore bleaching/blinking rates. For each curvature site of a POPC membrane, DPPETexas Red localization density at sites of curvature were in the range of $1-30 x$ higher than that of the planar bilayer, which is of significantly greater variation than observed via diffractionlimited imaging (Fig. 3). Therefore diffraction-limited images were used for phase-sorting studies.

\section{2) Membrane brightness at curvature sites}

The 1:1:2 SLBs phase sorting result indicated that the ratio of $P_{C} / P_{F}$ was greater than one at for all values of $P_{F}$ (Fig. 3). Thus, the disorder-preferring fluorescent lipid density was higher on curved membranes, which indicates that the $L_{d}$ phase sorts to curvature. The sorting was presumably due to the differences in the phase bending rigidity because the leaflets seemed to maintain phase registration (19) and we do not anticipate that the molecular shape (e.g., packing parameter) of the fluorescent lipid was sufficient to induce significant singlemolecule sorting (52-54). This is further supported by our observation that quasi-one component membranes of POPC were not able to support a curvature-induced sorting of fluorescent lipids.

The SLBs with molar ratios of 7:7:6 DOPC:DPPC:cholesterol with the fluorescent lipid TopFluor-PC also demonstrated phase sorting $\left(P_{C} / P_{F}>1\right)$, but only when the surrounding flat membrane was ordered $\left(P_{F}<1\right)$. Potentially there is less difference in the bending rigidity between the $L_{o}$ and $L_{d}$ phases in this system or more complex perturbations of the membrane shape are occurring.

\section{3) Distinguishing curvature from aggregation}

Distinguishing between lipid aggregates and curvature sites was critically important for proper data analysis. Upon single-fluorophore image reconstruction, the fluorescent lipid aggregates and the engineered curvature sites appeared similar in size and both had higher localization density than the planar bilayer. Curvature sites were always coincident with the fluorescent nanoparticle in the complementary color channel, and chromatic bleed through was confirmed to be negligible (30). If a lipid aggregate appeared within $300 \mathrm{~nm}$ with the fluorescent nanoparticle, then the sample was removed from further analysis. The diffusion rate of the lipids 
in the aggregates were $40 \%$ of $D_{F}$ and $50 \%$ of $D_{C}$. However, sample-to-sample variation caused difficulty precisely distinguishing aggregates versus curvature based on diffusion rates alone.

\section{4) Effects of the fluorescent lipid}

The lipid diffusion with nanoscale curvature varies with the fluorescence labeling strategy. For example, DPPE-Texas Red lipid diffusion in POPC bilayer in a curved membrane displayed a diffusion rate $40 \%$ of that of the flat membrane, $D_{C} / D_{F}=0.4 \pm 0.1$. However, TopFluor-PC showed no difference in diffusion with membrane bending, $D_{C} / D_{F}=1.0 \pm 0.2(26)$. DPPE-Texas Red was primarily used rather than TopFluor-PC throughout this manuscript because it was brighter, it could be used at lower concentrations, and it required less intense fluorescence illumination power. Moreover, tail-labeled fluorescent lipids perturb membrane more than head group-labeled fluorescent lipids as measured by transient binding versus free diffusion, which indicates the level of trapping interactions (55).

\section{5) Phase-dependent diffusion surrounding curvature sites}

Diffraction-limited images were used to assess the $P_{F}$ at $r=400 \mathrm{~nm}$ with $P_{F}<0.9$ indicating the $L_{o}$ phase and $P_{F}>1.1$ indicating the $L_{d}$ phase. This classification was used for sorting the curvature sites based on their surrounding lipid phase for diffusion analysis (Fig. 4). The $P_{F}$ values varied depending on $r$, which resulted in an uncertainty of the SLB phase and yielded no difference in the DPPE-Texas Red diffusion at $r=600 \mathrm{~nm}$. A more precise analysis of the effects of $P_{F}$ is shown in with diffraction-limited imaging when the strict classification of the phases was not necessary, and the value of $P_{F}$ surrounding each nanoparticle could be directly reported (i.e., Fig. 3). However, the diffusion analysis of Fig. 4 required combining the data multiple nanoparticles to yield meaningful results; thus, a binning of the $P_{F}$ values was necessary. A more robust analysis of the phase-dependent diffusion of fluorescent lipids is provided in our prior publication (24).

\section{5) $D_{C}>D_{F}$ in membranes with lipid mixtures}

The ratio of diffusion on curved versus flat membranes $\left(D_{C} / D_{F}\right)$ was larger when phase separation was present (Fig. 4, Table S1). This phenomenon was observed for all values of $P_{F}$, suggesting that the disorder-preferring lipids sorted to curvature even when the $L_{d}$ phase surrounded the curvature event. This result is consistent with sorting studies where $P_{C} / P_{F}$ was above 1 for all $P_{F}$ (Fig. 3). Whereas quasi-one component $L_{\alpha}$ membranes of DiPhyPC displayed no significant difference between $D_{C}$ and $D_{F}$, mixtures of DiPhyPC, cholesterol, and DPPC displayed faster diffusion at curved surfaces regardless of if phase separation was observed. We hypothesize that this demonstrates the capability of curvature to induce phase separation that is not present on flat membranes.

\section{6) Curvature-seeded phase separation propagates onto the surrounding flat membrane}

A subtle but consistent decrease in $D_{x y}$ was observed on the flat membrane propagating away from the engineered curvature sites. $D_{x y}$ decreased with increasing distance from the curvature for 125 to $400 \mathrm{~nm}$ away from the lateral center of the 50-nm radius nanoparticle (Fig. 4). This demonstrates that the effective membrane viscosity and the composition of the flat membrane varied with distance from the nanoparticle. We interpret this as a demonstration of the engineered curvature seeding phase separation that propagated onto the surrounding flat membrane. This phenomenon was also observed in simulations of phase separation with the $L_{d}$ phase more likely to be present on the flat membrane that is near the curvature (Fig. 6D).

\section{7) Alternative hypotheses}


Measuring the nanoscopic behaviors of lipids requires extracting the subtle signatures from the measurements. The data presented throughout this manuscript may be interpreted with alternative hypotheses that include varying leaflet phase registration, phase-dependent fluorophore brightness, and temperature-dependent membrane shape. A more thorough discussion of these possibilities is given in the Supplemental Materials.

\section{5) CONCLUSIONS}

Nanoscale membrane curvature was engineered in phase-separated supported lipid bilayers. The sorting and dynamics of lipids were measured to reveal the curvature-induced phase separation and the phase-curvature coupling. The intensity ratio of curvature and surrounding planar bilayer from diffraction-limited image shows the $L_{d}$ preferring lipids sorts to the engineered curvature. The single-lipid diffusion rates were measured as a function of distance away from the center of curvature to support the curvature-induced sorting of disordered lipids further. Unlike quasi-one component $L_{\alpha}$ phase membranes of DiPhyPC or POPC bilayer, SLBs with ternary mixtures of lipids showed a faster diffusion on the curvature than flat membranes. The effects of curvature on lipid diffusion were more pronounced under conditions in which the phase-separating tie-lines were longer, including lower temperatures and less cholesterol. These results indicate that the strong preference of disorder-preferring lipids at curvature sites and the capability of curvature inducing domains of greater disorder than are present on the planer membrane.

\section{6) AUTHOR CONTRIBUTIONS}

X.W. and C.V.K. designed the experiments and wrote the manuscript. X.W. performed the experiments and analyzed the data. C.V.K. performed the simulations.

\section{7) ACKNOWLEDGMENTS}

The authors thank Aurelia R. Honerkamp-Smith for valuable discussions. Financial support was provided by the Thomas C. Rumble University graduate fellowship, Wayne State University Summer Dissertation Award, and Richard J. Barber. This material is based upon work supported by the National Science Foundation under Grant No. DMR1652316. 


\section{8) ABBREVIATIONS}

$D_{C}$ : Diffusion coefficient on the curved membrane

$D_{F}$ : Diffusion coefficient on the flat membrane

$D_{x y}$ : Diffusion coefficient through the xy-plane

DiPhyPC: diphytanoylphosphocholine

DOPC: dioleoylphosphocholine

DPPC: dipalmitoylphosphatidylcholine

DPPE-Texas Red: dihexadecanoylphosphoethanolamine- Texas Red

FCS: fluorescent correlation spectroscopy

GUV: giant unilamellar vesicle

$<>$ : Averaged membrane intensity

$I_{C}$ : Fluorescence intensity of curved membrane

$I_{F}$ : Fluorescence intensity of flat membrane

$l_{d}$ : Fluorescence intensity of $L_{d}$ membrane

$I_{0}$ : Fluorescence intensity of $L_{0}$ membrane

$L:$ The homogeneous liquid lipid phase of a membrane with a mixture of lipids

$L_{\alpha}$ : The liquid lipid phase of a single-component membrane

$L_{d}$ : Liquid disordered phase

$L_{o}$ : Liquid ordered phase

MSD: mean square displacement

POPC: palmitoyloleoylphosphocholine

$P_{C}$ : Lipid phase of the curved membrane

$P_{F}$ : Lipid phase of the flat membrane

$r$ : distance away from the nanoparticle center

SLB: supported lipid bilayer

SPT: single-particle tracking

TopFluor-PC: TopFluor-palmitoylundecanoylphosphocholine 


\section{9) REFERENCES}

1. Pralle, A., P. Keller, E.-L. Florin, K. Simons, and J.K.H. Hörber. 2000. SphingolipidCholesterol Rafts Diffuse as Small Entities in the Plasma Membrane of Mammalian Cells. $J$ Cell Biol. 148:997-1008.

2. Simons, K., and E. Ikonen. 1997. Functional rafts in cell membranes. Nature. 387:569572.

3. Veatch, S.L., P. Cicuta, P. Sengupta, A. Honerkamp-Smith, D. Holowka, and B. Baird. 2008. Critical Fluctuations in Plasma Membrane Vesicles. ACS Chem. Biol. 3:287-293.

4. Fessler, M.B., and J.S. Parks. 2011. Intracellular lipid flux and membrane microdomains as organizing principles in inflammatory cell signaling. J. Immunol. 187:1529-1535.

5. Hurley, J.H., E. Boura, L.-A. Carlson, and B. Rózycki. 2010. Membrane Budding. Cell. 143:875-887.

6. Simons, K., and D. Toomre. 2000. Lipid rafts and signal transduction. Nat. Rev. Mol. Cell Biol. 1:31-39.

7. Waheed, A.A., and E.O. Freed. 2010. The Role of Lipids in Retrovirus Replication. Viruses. 2:1146-1180.

8. Parton, R.G., M.M. Kozlov, and N. Ariotti. 2020. Caveolae and lipid sorting: Shaping the cellular response to stress. J. Cell Biol. 219.

9. Parton, R.G., and K. Simons. 2007. The multiple faces of caveolae. Nat. Rev. Mol. Cell Biol. 8:185-194.

10. Kiessling, V., S.-T. Yang, and L.K. Tamm. 2015. Supported lipid bilayers as models for studying membrane domains. Curr Top Membr. 75:1-23.

11. Veatch, S.L., and S.L. Keller. 2002. Organization in lipid membranes containing cholesterol. Phys. Rev. Lett. 89:268101.

12. Veatch, S.L., and S.L. Keller. 2005. Seeing spots: Complex phase behavior in simple membranes. Biochimica et Biophysica Acta (BBA) - Molecular Cell Research. 1746:172185.

13. Wu, H.-M., Y.-H. Lin, T.-C. Yen, and C.-L. Hsieh. 2016. Nanoscopic substructures of raftmimetic liquid-ordered membrane domains revealed by high-speed single-particle tracking. Scientific Reports. 6:20542.

14. Baumgart, T., S.T. Hess, and W.W. Webb. 2003. Imaging coexisting fluid domains in biomembrane models coupling curvature and line tension. Nature. 425:821-824.

15. Sorre, B., A. Callan-Jones, J.-B. Manneville, P. Nassoy, J.-F. Joanny, J. Prost, B. Goud, and P. Bassereau. 2009. Curvature-driven lipid sorting needs proximity to a demixing point and is aided by proteins. Proc Natl Acad Sci U S A. 106:5622-5626. 
16. Heinrich, M., A. Tian, C. Esposito, and T. Baumgart. 2010. Dynamic sorting of lipids and proteins in membrane tubes with a moving phase boundary. PNAS. 107:7208-7213.

17. Tian, A., and T. Baumgart. 2009. Sorting of Lipids and Proteins in Membrane Curvature Gradients. Biophys J. 96:2676-2688.

18. Parthasarathy, R., C. Yu, and J.T. Groves. 2006. Curvature-modulated phase separation in lipid bilayer membranes. Langmuir. 22:5095-5099.

19. Ogunyankin, M.O., A. Torres, F. Yaghmaie, and M.L. Longo. 2012. Lipid domain pixelation patterns imposed by e-beam fabricated substrates. Langmuir. 28:7107-7113.

20. Chiantia, S., J. Ries, N. Kahya, and P. Schwille. 2006. Combined AFM and two-focus SFCS study of raft-exhibiting model membranes. Chemphyschem. 7:2409-2418.

21. Dietrich, C., L.A. Bagatolli, Z.N. Volovyk, N.L. Thompson, M. Levi, K. Jacobson, and E. Gratton. 2001. Lipid rafts reconstituted in model membranes. Biophys J. 80:1417-1428.

22. Ge, Y., J. Gao, R. Jordan, and C. Naumann. 2018. Changes in Cholesterol Level Alter Integrin Sequestration in Raft-Mimicking Lipid Mixtures. Biophysical Journal. 114:158-167.

23. Scherfeld, D., N. Kahya, and P. Schwille. 2003. Lipid Dynamics and Domain Formation in Model Membranes Composed of Ternary Mixtures of Unsaturated and Saturated Phosphatidylcholines and Cholesterol. Biophysical Journal. 85:3758-3768.

24. Woodward, X., and C.V. Kelly. 2020. Single-lipid dynamics in phase-separated supported lipid bilayers. bioRxiv. 2020.05.28.121830.

25. Domanov, Y.A., S. Aimon, G.E.S. Toombes, M. Renner, F. Quemeneur, A. Triller, M.S. Turner, and P. Bassereau. 2011. Mobility in geometrically confined membranes. Proc NatI Acad Sci U S A. 108:12605-12610.

26. Woodward, X., E.E. Stimpson, and C.V. Kelly. 2018. Single-lipid tracking on nanoscale membrane buds: The effects of curvature on lipid diffusion and sorting. Biochimica et Biophysica Acta (BBA) - Biomembranes. 1860:2064-2075.

27. Cheney, P.P., A.W. Weisgerber, A.M. Feuerbach, and M.K. Knowles. 2017. Single Lipid Molecule Dynamics on Supported Lipid Bilayers with Membrane Curvature. Membranes (Basel). 7.

28. Veatch, S.L. 2007. Electro-formation and fluorescence microscopy of giant vesicles with coexisting liquid phases. Methods Mol. Biol. 398:59-72.

29. Black, J.C., P.P. Cheney, T. Campbell, and M.K. Knowles. 2014. Membrane curvature based lipid sorting using a nanoparticle patterned substrate. Soft Matter. 10:2016-2023.

30. Kabbani, A.M., and C.V. Kelly. 2017. The Detection of Nanoscale Membrane Bending with Polarized Localization Microscopy. Biophysical Journal. 113:1782-1794.

31. Kabbani, A.M., and C.V. Kelly. 2017. Nanoscale Membrane Budding Induced by CTxB and Detected via Polarized Localization Microscopy. Biophysical Journal. 113:1795-1806. 
32. Baumgart, T., A.T. Hammond, P. Sengupta, S.T. Hess, D.A. Holowka, B.A. Baird, and W.W. Webb. 2007. Large-scale fluid/fluid phase separation of proteins and lipids in giant plasma membrane vesicles. Proc. Natl. Acad. Sci. U.S.A. 104:3165-3170.

33. Melby, E.S., A.C. Mensch, S.E. Lohse, D. Hu, G. Orr, C.J. Murphy, R.J. Hamers, and J.A. Pedersen. 2016. Formation of supported lipid bilayers containing phase-segregated domains and their interaction with gold nanoparticles. Environ. Sci.: Nano. 3:45-55.

34. Feigenson, G.W. 2009. Phase diagrams and lipid domains in multicomponent lipid bilayer mixtures. Biochim. Biophys. Acta. 1788:47-52.

35. Feigenson, G.W. 2006. Phase behavior of lipid mixtures. Nat. Chem. Biol. 2:560-563.

36. Ackerman, D.G., and G.W. Feigenson. 2015. Lipid Bilayers: Clusters, Domains and Phases. Essays Biochem. 57:33-42.

37. Heberle, F.A., and G.W. Feigenson. 2011. Phase Separation in Lipid Membranes. Cold Spring Harb Perspect Biol. 3.

38. Ovesný, M., P. Kř́žek, J. Borkovec, Z. Svindrych, and G.M. Hagen. 2014. ThunderSTORM: a comprehensive ImageJ plug-in for PALM and STORM data analysis and super-resolution imaging. Bioinformatics. 30:2389-2390.

39. Schindelin, J., I. Arganda-Carreras, E. Frise, V. Kaynig, M. Longair, T. Pietzsch, S. Preibisch, C. Rueden, S. Saalfeld, B. Schmid, J.-Y. Tinevez, D.J. White, V. Hartenstein, K. Eliceiri, P. Tomancak, and A. Cardona. 2012. Fiji: an open-source platform for biologicalimage analysis. Nat. Methods. 9:676-682.

40. Jaqaman, K., D. Loerke, M. Mettlen, H. Kuwata, S. Grinstein, S.L. Schmid, and G. Danuser. 2008. Robust single-particle tracking in live-cell time-lapse sequences. Nat Meth. 5:695-702.

41. Cheney, P.P., A.W. Weisgerber, A.M. Feuerbach, and M.K. Knowles. 2017. Single Lipid Molecule Dynamics on Supported Lipid Bilayers with Membrane Curvature. Membranes (Basel). 7.

42. Knight, J.D., M.G. Lerner, J.G. Marcano-Velázquez, R.W. Pastor, and J.J. Falke. 2010. Single Molecule Diffusion of Membrane-Bound Proteins: Window into Lipid Contacts and Bilayer Dynamics. Biophysical Journal. 99:2879-2887.

43. Berglund, A.J. 2010. Statistics of camera-based single-particle tracking. Phys. Rev. E. 82:011917.

44. Lagerholm, B.C., D.M. Andrade, M.P. Clausen, and C. Eggeling. 2017. Convergence of lateral dynamic measurements in the plasma membrane of live cells from single particle tracking and STED-FCS. J Phys D Appl Phys. 50:063001.

45. Qian, H., M.P. Sheetz, and E.L. Elson. 1991. Single particle tracking. Analysis of diffusion and flow in two-dimensional systems. Biophys J. 60:910-921. 
46. Kabbani, A.M., X. Woodward, and C.V. Kelly. 2017. Revealing the Effects of Nanoscale Membrane Curvature on Lipid Mobility. Membranes. 7:60.

47. Sadeghi, S., M. Müller, and R.L.C. Vink. 2014. Raft Formation in Lipid Bilayers Coupled to Curvature. Biophysical Journal. 107:1591-1600.

48. Gunderson, R.S., and A.R. Honerkamp-Smith. 2018. Liquid-liquid phase transition temperatures increase when lipid bilayers are supported on glass. Biochimica et Biophysica Acta (BBA) - Biomembranes. 1860:1965-1971.

49. Bag, N., D.H.X. Yap, and T. Wohland. 2014. Temperature dependence of diffusion in model and live cell membranes characterized by imaging fluorescence correlation spectroscopy. Biochimica et Biophysica Acta (BBA) - Biomembranes. 1838:802-813.

50. Sengupta, P., A. Hammond, D. Holowka, and B. Baird. 2008. Structural Determinants for Partitioning of Lipids and Proteins Between Coexisting Fluid Phases in Giant Plasma Membrane Vesicles. Biochim Biophys Acta. 1778:20-32.

51. Tamm, L.K. 1988. Lateral diffusion and fluorescence microscope studies on a monoclonal antibody specifically bound to supported phospholipid bilayers. Biochemistry. 27:14501457.

52. Callan-Jones, A., B. Sorre, and P. Bassereau. 2011. Curvature-Driven Lipid Sorting in Biomembranes. Cold Spring Harb Perspect Biol. 3.

53. Cooke, I.R., and M. Deserno. 2006. Coupling between Lipid Shape and Membrane Curvature. Biophys J. 91:487-495.

54. Kamal, M.M., D. Mills, M. Grzybek, and J. Howard. 2009. Measurement of the membrane curvature preference of phospholipids reveals only weak coupling between lipid shape and leaflet curvature. PNAS. 106:22245-22250.

55. Mobarak, E., M. Javanainen, W. Kulig, A. Honigmann, E. Sezgin, N. Aho, C. Eggeling, T. Rog, and I. Vattulainen. 2018. How to minimize dye-induced perturbations while studying biomembrane structure and dynamics: PEG linkers as a rational alternative. Biochimica et Biophysica Acta (BBA) - Biomembranes. 1860:2436-2445.

56. Blosser, M.C., A.R. Honerkamp-Smith, T. Han, M. Haataja, and S.L. Keller. 2015. Transbilayer Colocalization of Lipid Domains Explained via Measurement of Strong Coupling Parameters. Biophys. J. 109:2317-2327. 


\section{SUPPLEMENTAL MATERIAL}

\section{S.1) Identification of aggregates}

Super-resolution images reconstructed from single-fluorophore localizations contained aggregates of DPPE-Texas Red in the DiPhyPC-containing membranes. The clusters of these dense, aggregate localizations were $63 \pm 17 \mathrm{~nm}$ radius and showed confinement in lipid diffusion. DiPhyPC contains phytanoyl acyl tails that are highly disordered and have reduce photo-oxidization compared to unsaturated lipids. Photo-stability is especially appreciated in experiments that rely on phase separations that are sensitive to the membrane composition.

Aggregates were identified and removed by custom algorithms that were studied extensively previously (24). Aggregates were removed via binning all localizations into voxels of space and acquisition time and culling based on a threshold localization rate. When the number of localizations in a voxel exceeded the localization density threshold $\left(\rho_{t h}\right)$, that region of the sample was deemed to be an aggregate, and all localizations within that $\mathrm{XY}$-region were excluded from subsequent analysis. Further, all localizations associated with trajectories that lasted more than 32 steps were culled. The value of $\rho_{\text {th }}$ was varied such that between 0 and 80 percentiles of the total localizations were culled. For each $\rho_{t h}$, a simulated membrane was generated with an evenly distributed localizations except the removed region. A spatial correlation function was calculated for both the experimental and simulated membrane and the remaining localizations according to

$$
g(r)=\frac{\left\langle F F T^{-1}\left(|F F T(I(\vec{r}))|^{2}\right)\right\rangle_{\theta}}{\rho^{2}}
$$

$I(\vec{r})$ represents the two-dimensional localization histogram, and $\rho$ is the average localization density. The average number of localizations per cluster can be calculated by comparing the spatial correlation of the two.

$$
N=\int\left(\frac{g_{\text {exp }}}{g_{\text {sim }}}-1\right) r d r
$$

$N$ was calculated for all localization data sets with varying $\rho_{t h}$. The minimum $\rho_{t h}$ for which $\mathrm{N} \leq 3$ was used for the diffusion studies.

\section{S.2) Alternative hypotheses}

\section{Leaflet registration}

The analysis performed throughout this manuscript assumes that the lipid phases are registered such that there are only two levels of bilayer brightness across a phase-separated bilayer. The interplay between leaflets is sufficiently great that supported lipid bilayers only display deregistration of a shear force is applied (56). If the phases became deregistered between the top and bottom leaflets, then three different levels of brightness would be observed. Although three distinct brightness levels were never observed on the flat bilayer, neither the super-resolution nor diffraction-limited imaging performed here was able to resolve subtle brightness variations across the $50-\mathrm{nm}$ radius nanoparticle-supported membrane curvature. Because we have no data to the contrary, our assumption throughout this manuscript assumes that the phases-maintained registration on the curved membrane.

However, phase deregistration may be expected in other systems in which a phasedependent spontaneous curvature was expected. For example, if the $L_{d}$ phase preferred 
negative curvature and the $L_{o}$ phase preferred positive curvature, this would couple with the engineered bilayer shape to encourage phase deregistration.

\section{Consistent fluorophore brightness}

The local diffraction-limited fluorescence emission intensity of the DPPE-Texas Red was used as a measure of the local concentration of DPPE-Texas Red. This analysis assumed that each fluorophore was consistently bright regardless of the lipid phase or membrane curvature. The DPPE-Texas Red molecules in the varying lipid phases or curvature may have varying quantum efficiency or self-quenching that complicated the connection between fluorescence emission intensity and DPPE-Texas Red concentration. Accordingly, the measurements of the lipid phase performed here that are based on the fluorescence brightness may require a rescaling to quantitatively connect to other measurement methods that are free from this complication (i.e., NMR).

\section{Consistent membrane shape}

The membrane curvature in this study was engineered by draping SLBs over $50-\mathrm{nm}$ radius polystyrene nanoparticles. The degree to which the membrane either conformally wraps around the nanoparticle to make a narrow neck attaching the membrane bud to the planar membrane or treats the nanoparticle more of a tent pole and lifts off the coverslip is not known. Assumptions were made in this manuscript and previously $(24,30,46)$ that the SLB maximizes its contact with the supporting substrate while never bending to a tighter than a $20-\mathrm{nm}$ radius of curvature. This assumed structure yields consistent simulations and experimental data for varying nanoparticle diameters and diffusion rates. Throughout this manuscript, the membrane shape was assumed to be constant for varying membrane compositions, temperature, and phase.

\section{Leaflet-dependent diffusion and the effects of the varying substrate material}

The nanoscale membrane curvature is generated over polystyrene nanoparticles. Polystyrene and glass have different surface properties and effects on the SLBs supported by them. We performed control experiments to confirm that the curvature-dependent diffusion was not due to the varying curvature or substrates material properties (26). Namely, we created SLBs with the fluorescent lipids either in both leaflets or only in the top leaflet, far from the substrate. The diffusion in the top leaflet was faster than that of the bottom leaflet; however, the effects of curvature were indistinguishable on the leaflets. 


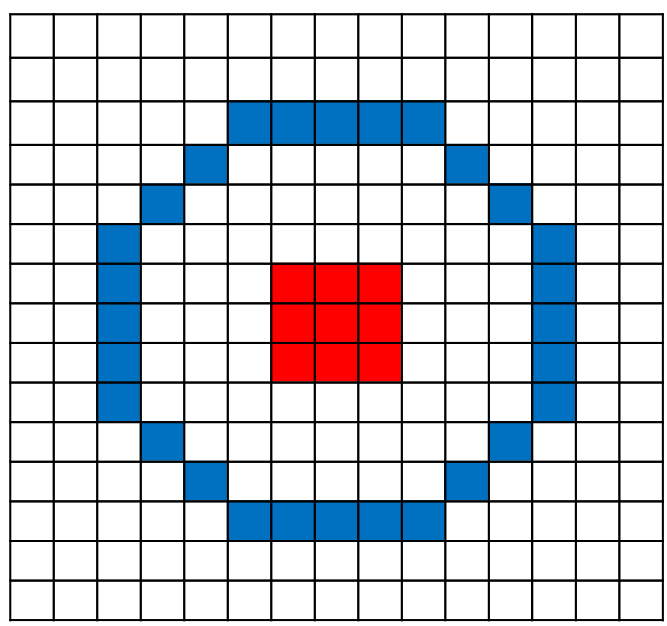

Figure S1: To measure the sorting of lipids to the curvature, the center 9 pixels (red) were deemed to be equal to the curved membrane and the pixels that were closest to $400 \mathrm{~nm}$ from the nanoparticle center were deemed equal to the surrounding flat membrane (blue). Each pixel maps to $80 \mathrm{~nm} \times 80 \mathrm{~nm}$ of our sample.
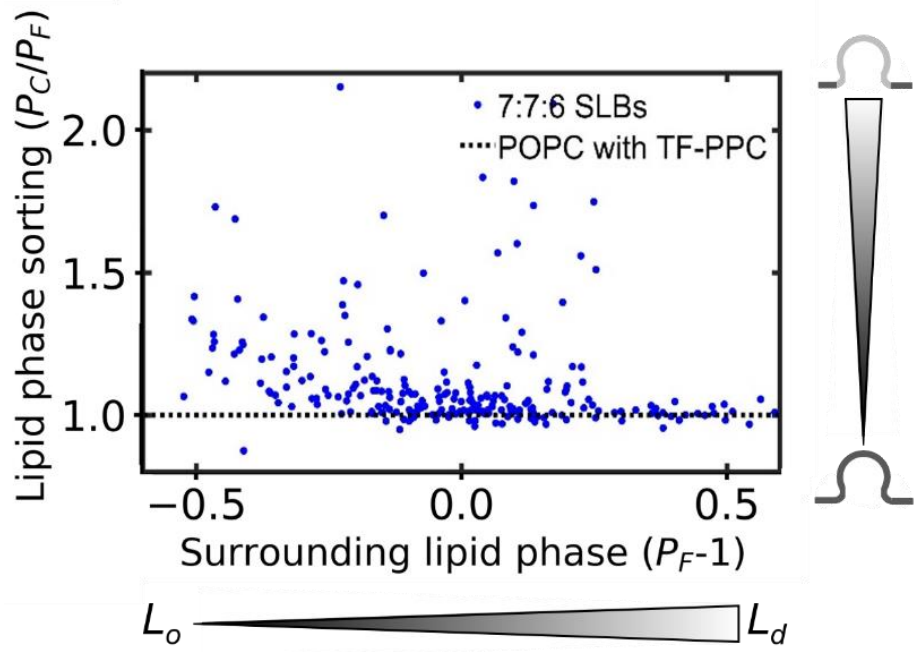

Figure S2: Phase sorting as function of surrounding phase for 7:7:6 DOPC:DPPC:cholesterol SLBs. The TopFluor-PC are less accumulated at curvature sites even in POPC membrane. Unlike 1:1:2 SLBs, 7:7:6 SLBs showed no sorting to curvature at large $P_{F}$ 

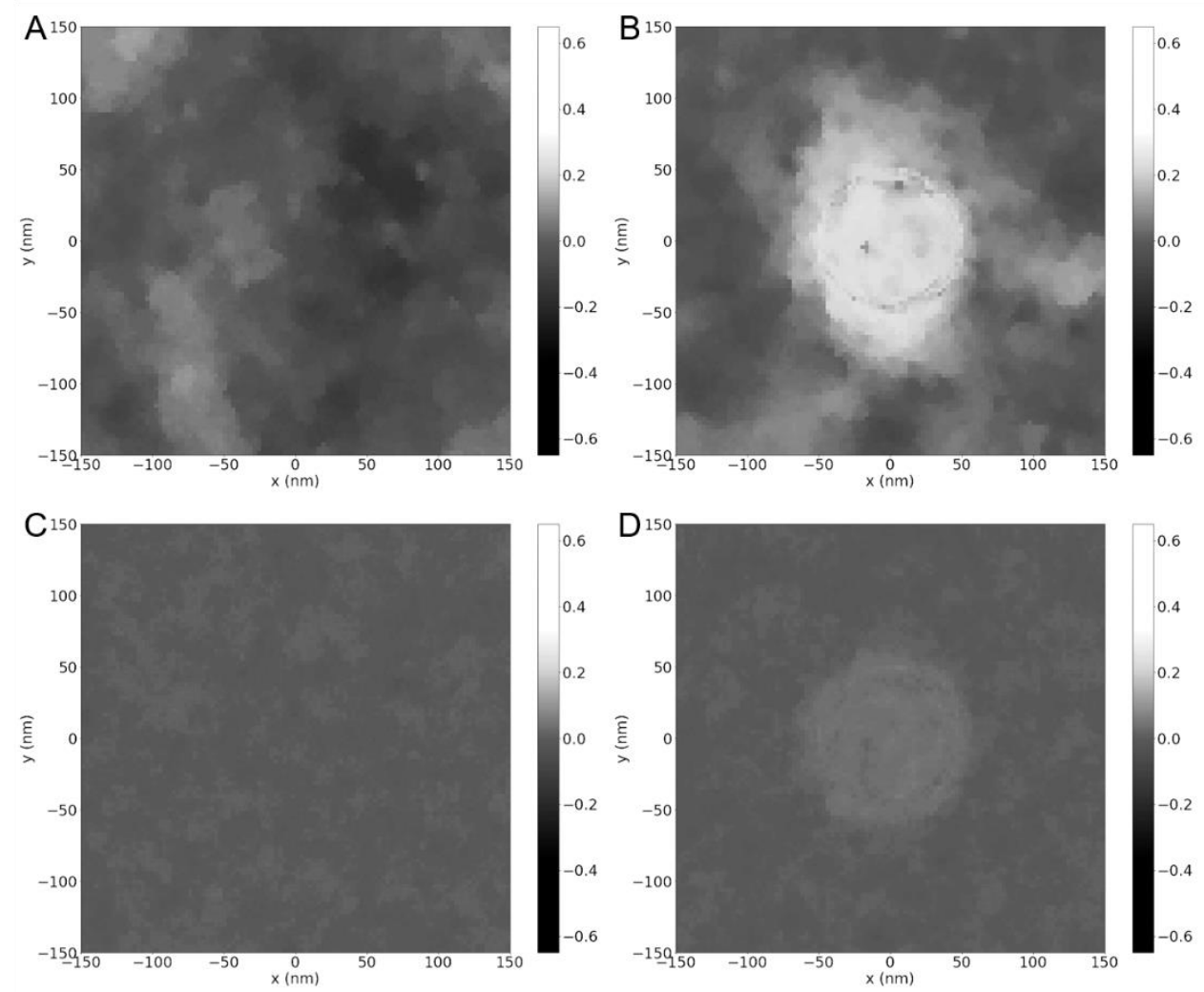

Figure S3: The time-average simulation result for the equilibrated membrane phase separations and fluctuations relative to membrane curvature. These simulations included $(A, B)$ cold $(\sigma=0.07)$ and $(C, D)$ hot $(\sigma=0.08)$ membranes that are $(A, C)$ flat or $(B, D)$ with a $50-\mathrm{nm}$ radius membrane bud with a phasecurvature coupling $(\gamma)$ of 0.1 . These data are the same as those shown in Fig. 6 and Movie 1. 
Table S1: Diffusion coefficient results for varying membrane compositions, temperatures, and phases. The $D_{F}$ uncertainties are from the standard deviation $D_{x y}$ values for $r$ between 400 and $600 \mathrm{~nm}$. The $D_{C} / D_{F}$ and uncertainties are from the propagated error of $D_{x y}(r<50 \mathrm{~nm})$ fitting and $D_{F}$

\begin{tabular}{cccccc}
\hline Composition & $\mathbf{T}\left({ }^{\circ} \mathbf{C}\right)$ & Phase & \# of samples & $\boldsymbol{D}_{\boldsymbol{F}}\left(\boldsymbol{\mu} \boldsymbol{m}^{2} / \boldsymbol{s}\right)$ & $\boldsymbol{D}_{\boldsymbol{C}} / \boldsymbol{D}_{\boldsymbol{F}}$ \\
\hline POPC & 25 & $L_{\alpha}$ & 12 & $2.6 \pm 0.03$ & $0.4 \pm 0.1$ \\
$1: 0: 0$ & 17 & $L_{\alpha}$ & 19 & $1.03 \pm 0.03$ & $1.2 \pm 0.4$ \\
$1: 0: 0$ & 36 & $L_{\alpha}$ & 18 & $1.75 \pm 0.03$ & $0.7 \pm 0.4$ \\
$1: 1: 2$ & 19 & $L_{o}$ & 8 & $0.78 \pm 0.04$ & $2.3 \pm 1.7$ \\
$1: 1: 2$ & 19 & $L_{d}$ & 8 & $0.74 \pm 0.13$ & $2.1 \pm 1.4$ \\
$1: 1: 2$ & 27 & $L_{o}$ & 14 & $1.27 \pm 0.09$ & $1.5 \pm 0.5$ \\
$1: 1: 2$ & 27 & $L_{d}$ & 8 & $1.28 \pm 0.07$ & $1.3 \pm 0.4$ \\
$1: 1: 2$ & 37 & $L$ & 11 & $1.44 \pm 0.09$ & $1.4 \pm 0.5$ \\
$2: 2: 1$ & 17 & $L_{o}$ & 11 & $0.67 \pm 0.04$ & $1.7 \pm 0.7$ \\
$2: 2: 1$ & 17 & $L_{d}$ & 14 & $0.57 \pm 0.06$ & $2.3 \pm 1.0$ \\
$2: 2: 1$ & 27 & $L_{o}$ & 10 & $1.19 \pm 0.06$ & $1.1 \pm 0.7$ \\
$2: 2: 1$ & 27 & $L_{d}$ & 16 & $1.37 \pm 0.03$ & $1.5 \pm 0.6$ \\
\hline
\end{tabular}

